\title{
Encartes
}

ISSN 2594-2999, Bajo licencia Creative Commons

encartesantropologicos@ciesas.edu.mx

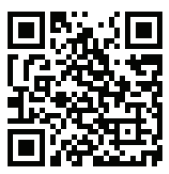

Aceves Lozano, Jorge E.

Culturas Ciudadanas y Ciudadanía Cultural. Una exploración de los términos

Encartes, vol. 3, núm. 6, septiembre 2020-febrero 2021, pp. 161-187

Enlace: https://doi.org/10.29340/en.v3n6.116

Jorge Eduardo Aceves Lozano ORCID: 0000-0002-8488-4610

Disponible en https://encartesantropologicos.mx/ 


\section{REALIDADES SOCIOCULTURALES}

\section{GULTURAS GIUDADANAS Y}

GIUDADANÍA GULTURAL. UNA EXPLORAGIÓN DE LOS TÉRMINOS

GITIZEN GULTURES AND GULTURAL GITIZENSHIP.

AN EXPLORATION OF THE TERMS

Jorge E. Aceves Lozano*

Resumen: En este texto se hace una revisión acerca de la relación entre ciudadanía y cultura. Explorar textos de ciencias sociales que tratan de estos términos para analizar determinados sujetos sociales, tanto en su acción como en su conceptualización, nos lleva a considerar que las ciudadanías son diversas, heterogéneas y con posiciones desiguales respecto a otros conjuntos de ciudadanos/as y en su relación con la esfera estatal. Cada conjunto de ciudadanos/as vive y modela su acción social con base en sus propias configuraciones identitarias, códigos y disposiciones culturales, afectados todos ellos por relaciones de poder y género, de clase y etnia. Las ciudadanías expresan -a través de sus acciones, emociones y pensamientos- la pluralidad social, política, económica y cultural de nuestras conflictivas sociedades contemporáneas. Dos reflexiones se desarrollan en este ensayo: primero se aborda la discusión desde las prácticas ciudadanas; en una segunda mirada se resalta la dimensión cultural que dichas prácticas manifiestan de derechos ciudadanos específicos.

Palabras claves: ciudadanía, cultura, política, derechos, globalización.

GITIZEN GULTURES AND GULTURAL GITIZENSHIP. AN EXPLORATION OF THE TERMS

Abstract: This text reviews the relationship between citizenship and culture. Exploring social science literature on these terms to analyze particular social subjects, both in their action and their conceptualization, has led us to consider that citizenships are diverse, heterogenous and with unequal positions in regard to other citizens and in their relationship with the sphere of the State. Each

* Centro de Investigaciones y Estudios Superiores en Antropologia Social Occidente.

ISSN 2594-2999, Bajo licencia Creative Commons

Encartes 6 •septiembre 2020-febrero 2020, pp. 161-187

Recepción: 27 de mayo de 2019 • Aceptación: 10 de octubre de 2020

http://www.encartesantropologicos.mx

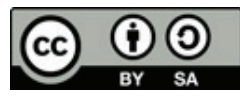


group of citizens lives and models its social action based on its own identity configurations, codes and cultural dispositions, all of which are affected by power, gender, class and ethnicity relations. Citizenships express -through their actions, emotions, and thoughts - the social, political, economic and cultural diversity of our contemporary conflictive societies. Two considerations develop in this text: first, the discussion is addressed from citizen practices; a second look highlights the cultural dimension that these practices express about specific citizen rights.

Keywords: citizenship, culture, politics, rights, globalization.

a RElaGión CiUdAdanía $(\mathrm{s})+$ GULTURA $(\mathrm{s})$
Comenzar por distinguir entre ciudadanía y cultura es un paso necesario pero incierto, ya que ambos términos conllevan definiciones polisémicas, cuyo dinamismo en su caracterización ha sido evidente en los últimos años entre los analistas que indagan sobre los procesos de cambio sociocultural. No será lo mismo iniciar con la más conocida combinación de los conceptos de la llamada "cultura ciudadana" que con una más reciente alternativa teórico-metodológica para la descripción y el análisis de la ciudadanía en su dimensión cultural (Miller, 2002; Hermes, 2006; Nivón, 2014).

No se reduce esta distinción a un orden de los factores conceptuales, sino que remite a posicionamientos y prioridades en la concepción de la realidad social y los modos de conocerla e intervenir en ella. De igual manera, añadir a cada concepto la cualidad plural modifica la connotación asignada, de allí que referirse a "culturas" y a "ciudadanías" añade una cualidad apenas perceptible, pero que de modo cierto incide en la relativa dureza de las definiciones a priori, cerradas y estáticas, por no decir limitadas. Esto nos conduce a la afirmación y al supuesto teórico de que las ciudadanías son diversas, heterogéneas y en posiciones desiguales respecto de otros conjuntos de ciudadanos/as y en su relación con la esfera estatal. Cada conjunto de ciudadanos/as vive y modela su acción social con base en sus propias configuraciones identitarias y disposiciones culturales, afectadas todas ellas por relaciones de poder y género, de clase y etnia. Las ciudadanías expresan la pluralidad social, política, económica y cultural de nuestras sociedades contemporáneas (Heater, 2007; Emmerich, 2009; Escalante, 2014). De igual forma, expresan sus contradicciones, sus vicios y miedos, sus limitaciones y obstáculos; no sólo el lado luminoso de la ac- 
ción creadora, positiva, también la faz negativa, oscura y retrógrada de la vida social.

La acción colectiva de las ciudadanías tiene múltiples dimensiones (política, social, económica), no obstante, la cultural es una que ha cobrado relevancia para el análisis de éstas, ya que funciona como plataforma orientadora de la acción ciudadana. Los modelos de participación en la vida social, los sistemas de creencias, los argumentos sobre el orden social y las concepciones acerca del mundo y el papel que juegan en él son fórmulas, esquemas y mapas orientadores del pensar y hacer del accionar ciudadano. Acciones que se desarrollan tanto en espacios públicos convencionales o bien en nuevos espacios públicos como las redes sociales en Internet, donde también es posible ejercitar la ciudadanía. Espacios públicos diversos que posibilitan una función convivencial, integradora y expresiva de las aspiraciones ciudadanas en el ejercicio y la reivindicación de sus derechos (Borja, 2014: 239-242) y que por lo general se objetivan en específicos territorios de paisajes urbanos/rurales en procesos que no logran siempre consolidarse del todo.

El léxico en torno a las cualificaciones de la ciudadanía es extenso; la mayor parte de los términos presentan ampliaciones de los derechos existentes y algunos más son proyectos en potencia y en cierta medida asociados a un horizonte utópico, pero sin duda orientadores y animadores de la acción de ciudadanías activas. En el siguiente listado podremos indagar definiciones y argumentos sobre las nuevas y diversas categorías de ciudadanías. Ciudadanía cultural (Rosaldo, 1994 y 2000; Kymlicka, 1996; unesco, 1999; García Canclini,1999; Safa, 1999 y 2001; Bonilla, 1999; Turner, 2001; Calderón, Assies y Salman, 2002; Joppke, 2002; Miller, 2002; Hermes, 2006; Nivón, 2014; Florescano y Cossío, 2014; Gavilán, 2018). Ciudadanía étnica (Calderón, Assies y Salman, 2002; Kabeer, 2007; De la Peña, 2008; Garzón, 2010; Cerda et al., 2011; Gerda, 2012; Ortiz, 2012). Ciudadanía digital/mediática (Winocur, 2002; Pérez Luño, 2003; Hermes, 2006; Galindo, 2009; Robles, 2009; Merino y Vega, 2011; Natal, Benítez y Ortiz, 2014; Adame, 2015; Richter, 2018). Ciudadanía mundial/global (Cortina, 1997; García Canclini, 2001; Caletti, 2003; Ramírez Saiz, 2006 y 2014). Ciudadanía cosmopolita (Hannerz, 1998; Linklater, 2002 y 2007; Norris, 2005; Aguilera, 2010; Abrahamian, 2015). Ciudadanía juvenil/infantil (Muñoz, 2008; Padilla y Flores, 2011; Earls, 2011; Reguillo, 2003 y 2017). Ciudadanía ecológica (Steenbergen, 1994; Riechmann y Fer- 
nández, 1994; Aceves, 1997 y 2011; Curtin, 2002; Aguilera, 2010). Ciudadanía precaria (Moreno, 2000; Caletti, 2003). Ciudadanía neoliberal (Kabeer, 2007; Zamorano, 2008). Ciudadanía moderna (Turner, 1994; Kabeer, 2005; Zamorano, 2008). Ciudadanía múltiple (Mateos, 2015). Ciudadanía inmigrante (Ansley, 2007; Rubio, 2010; Hernández López et al., 2018) Ciudadanía de género y sexual (González Luna, 1997; Richardson, 2001; Lister, 2002). Ciudadanía vecinal (Safa, 1999). Ciudadanía flexible (Ong, 2008). Ciudadanía incluyente (Kabeer, 2005). Ciudadanía amplia (Calderón, Assies y Salman, 2002; Aceves, 2011; Hernández González, 2015). Ciudadanía democrática (Rubio, 2007). Ciudadanía multilateral (Santiago, 2012); Ciudadanía activa (Lechner, 2000). Ciudadanía emergente (Jelín, 1994; Isin y Turner, 2002; Reigadas y Cullen, 2003). Aunque no pretende ser una lista exhaustiva, nos permite dar cuenta de una variedad pluritemática y de campos de acción de configuraciones ciudadanas dispersas a lo largo del planeta y que se han desarrollado particularmente desde los años ochenta del siglo xx.

\section{Primera mirada: GiUdAdanía $\div$ PrÁcticas GUlTurales $=$ GULTURAS GIUDADANAS}

Sin caer en la definición simplista de concebir a la cultura como una expresión del folklore de un pueblo en particular ni reducir su contenido a los patrones expresivos de las bellas artes y los modos cultos de la creatividad humana, convendría tomar una definición de cultura más compleja y de carácter antropológico (Nivón, 2014), que básicamente se definirá como la configuración de un conjunto de símbolos, concepciones y prácticas significativas interrelacionadas que estructuran y dinamizan a una sociedad, en un espacio y contexto sociohistórico determinados. El marco amplio del ejercicio ciudadano será entonces el de la actual sociedad de la información, la comunicación y el conocimiento. Las culturas se moverán en un contexto de mundialización, o si se prefiere, de la globalización impulsada por la transformación y reestructuración del capitalismo avanzado (García Canclini, 1999; Linklater, 2002 y 2007; Castells, 2000 y 2009).

Estos cambios globales acelerados a partir de los años setenta y ochenta han propiciado el surgimiento y desarrollo de un gran número de grupos e instituciones de ciudadanos/as de muy diversa índole, que ejercitan su quehacer político en las distintas dimensiones de la realidad social (política, económica y cultural) y no sólo frente al Estado, también frente a sus conciudadanos y las diversas comunidades emergentes, a partir de la 
acción colectiva. La actuación ciudadana toma formas plurales de expresión, ya sea como sociedad civil organizada, o bien en formas concretas de asociaciones civiles (OSC), organismos no gubernamentales (ONG), organizaciones del tercer sector, las no lucrativas y otras tantas formas concretas de acción ciudadana no convencionales, es decir alejadas de los organismos sindicales o de partidos políticos, por ejemplo

Las culturas ciudadanas y sus modos específicos de concreción implican un conjunto de elementos que aportan a su configuración: la forma en que nos concebimos a nosotros mismos como ciudadanía, las imágenes que tenemos sobre la manera de vivir e interactuar en nuestra sociedad; la responsabilidad que tenemos frente a los demás miembros de la sociedad y viceversa, lo que esperamos de los otros en su relación con nosotros (Arredondo, 1996). La "cultura ciudadana" - en singular- es el conjunto de valores, motivaciones y conductas que ejercitamos cotidianamente en nuestras interrelaciones en los contextos sociales que vivimos. Aunque se concibe que la cultura ciudadana es la forma en que se entienden y se ejercitan los derechos y obligaciones al formar parte de una comunidad particular, no sería conveniente reducir su alcance a esta acotación definitoria (Valderrama, 2007). Se vislumbra la oportunidad histórica de enfatizar la acción ciudadana más hacia la dimensión de los deberes que a la de los derechos ciudadanos (Arredondo, 2000: 16), donde la acción colectiva construye en el diálogo y la confrontación los nuevos perfiles de ciudadanías, que ya no estarán sólo enmarcadas y contenidas por la lógica de la adscripción y la concesión del reconocimiento de los derechos por parte del Estado.

Se argumenta así que las culturas ciudadanas son los modos plurales de ejercitarse colectivamente en los asuntos que competen al individuo y a su entorno local, sin dejar de lado los problemas más relevantes del contexto nacional y aun del internacional. Es además un ejercicio colectivo, no sólo un acto individualista, que se modela y orienta en las prácticas de interacción con otros actores sociales, no sólo referido a la esfera de la política sino al más amplio espectro de la estructura social. Las culturas ciudadanas se despliegan y están ubicadas en contextos sociohistóricos determinados, por lo que son dinámicas, se adaptan y reconfiguran según patrones de interacción y posibilidades de actuación. Están enmarcadas y afectadas por las circunstancias y los acontecimientos del mundo de lo cotidiano, con expectativas y horizontes de actuación que se sostienen en las relaciones 
cara a cara y en los contextos que limitan o expanden sus capacidades de interacción. En los contextos políticos de sociedades democráticas como la que limitadamente tenemos en nuestro país, no puede quedar reducida la concepción de la democracia al mero hecho de operar procesos electorales para definir las elites políticas y los liderazgos formales. Las iniciativas ciudadanas requieren de una democracia menos formal y más alternativa, que exprese un proceso vivo de acción ciudadana, de construcción e imaginación política. Las culturas ciudadanas se enfrentan así a tradiciones y modos convencionales de ejercer la democracia y de participar en la vida política de la sociedad (Rodríguez, 2005: 13-15). Cuando se piensa en los procesos de formación de ciudadanía como requisito para mejorar la calidad de la vida democrática, el término de cultura ciudadana se torna en acción política, en tanto que se le concibe y describe, en palabras de Antanas Mockus (exalcalde de Bogotá) como "el conjunto de costumbres, acciones y reglas mínimas compartidas que generan sentido de pertenencia, facilitan la convivencia urbana y conducen al respeto del patrimonio común y al reconocimiento de los derechos y deberes ciudadanos" (citado en Escobedo y Camargo, 2006: 92); un valioso conjunto de ideas que han nutrido políticas públicas en distintas geografías de Latinoamérica. Políticas públicas de cultura ciudadana, que buscan "transformar comportamientos específicos de la ciudadanía”, y deben contener un ejercicio de focalización e intervención sistemática en problemáticas que afectan la vida en comunidad (Mockus et al., 2012: 26) con el fin de impulsar el bienestar de la ciudadanía y la democratización de las ciudades.

La ciudadanía como expresión de una práctica social, como un ejercicio de construcción, se opone a la definición de un sujeto pasivo dueño de ciertos derechos y cumplidor acrítico de unos deberes (Krotz y Winocur, 2007). La potencialidad de la ciudadanía al desplegar su iniciativa es una parte central de la vida en democracia, en tanto que evidencia la capacidad de ser sujeto del proceso de su desarrollo humano. Esta agencia se manifiesta en su libertad para elegir y determina los fines de su acción, tanto en el proceso de intervenir sobre la organización de lo social como en lo político.

Norbert Lechner (2015) expresaba que la calidad de la democracia estaba asociada en buena medida con cuán democrática era la convivencia social, cuestión que dependía de los contextos sociales, los mapas mentales o códigos de interpretación disponibles que orienten al ciudadano a de- 
finir las alternativas de acción. Para que la ciudadanía pudiera lograr ser efectivamente sujeto actuante (individual y colectivo), dispondría de un conjunto de capacidades sociales y derechos básicos que contribuyeran a sostener su acción y lograr sus fines imaginados. De manera tal que cuanto mayor fueran las capacidades de ser sujeto, mejores serían las condiciones y posibilidades de la acción ciudadana.

Pero ¿a qué capacidades se refería para potenciar la acción ciudadana? Eran cinco: a) capacidades organizativas, b) capacidades cognitivas para determinar lo posible, c) capacidades morales en el sentido de un marco normativo, d) capacidad de simbolizar las relaciones sociales, y e) capacidad de establecer una relación emocional y afectiva con la democracia. Sin embargo, no será fácil desarrollarlas, tomando en cuenta los obstáculos de los actuales contextos sociales, económicos, políticos y culturales que no sólo limitan su creación, sino más aún su potenciación (Lechner, 2015: 319-325). Además, asentaba que la ciudadanía tiene que ver con la fortaleza del vínculo social, pues a falta de ésta la desavenencia política prevalecerá (Castel, 2010). Por lo que fortalecer la ciudadanía implica potenciar la vida social y la democratización de la sociedad, expandir el ámbito público en el que se favorezcan la multiplicación de lazos de confianza y la cooperación cívica, espacios que permitan la posibilidad de compartir experiencias por ámbitos de conversación y encuentros ciudadanos. Lo cual no dependerá de una política institucional, sino y en consideración de la iniciativa y acción ciudadanas (Lechner, 2000: 27-28).

Como sujeto históricamente contextualizado, la ciudadanía se adscribe también a culturas concretas igualmente identificables, a universos simbólicos desde los cuales se legitiman normativa y cognoscitivamente su vínculo y su relación significativa con el mundo que los rodea (Valderrama, 2007: 220). Esto conduce también a pensar que la pertenencia del sujeto a una comunidad no es sólo por ser comunidad política sino por ser comunidad de comunicación, o lo que también se nombra como de interpretación compartida. Las culturas ciudadanas orientan la comunicación intercultural en el sentido de impulsar procesos de comprensión de las convergencias y divergencias entre las interpretaciones que personas de culturas diferentes atribuyen a ciertos acontecimientos o procesos sociales. El ejercicio de la ciudadanía, apunta Valderrama, se encamina de esta manera a operar como una empresa hermenéutica, donde se decodifican e interpretan determinados lenguajes, prácticas y símbolos (2007: 221). 
Como ejercicio de tal comunicación e interpretación, las nuevas culturas ciudadanas imaginan y configuran espacios públicos inéditos que exploran formas nuevas de comunicación, información y conocimiento, que producen distintas y a veces contradictorias prácticas culturales. Por ejemplo, las esferas públicas virtuales, los espacios de los ciudadanos/ as desarrollados en internet (redes sociales, foros temáticos de discusión, chats y plataformas de información/vinculaciones diversas). El surgimiento y desarrollo de estos nuevos espacios comunicativos produce las condiciones tecno-simbólicas para el ejercicio ciudadano en una esfera pública de alcance global, de gran autonomía, con operación y flujo de información permanente, relativamente desterritorializada y desanclada de las censuras y exclusiones de espacios políticos convencionales (Castells, 2012; Natal et al., 2014; Abrahamian, 2015; Reguillo, 2017). La ampliación del ejercicio de la ciudadanía se potencia al tener acceso a estos nuevos escenarios globales de comunicación, lo que no significa el olvido de las plataformas previas, como lo ha sido regularmente la radio (Winocur, 2002). No obstante, este acceso continúa siendo desigual, con usos contradictorios y efectos perniciosos, y no es democrático en relación con la posibilidad de acción de la mayor parte de la ciudadanía. Disminuir la llamada brecha digital, o sea la exclusión en el acceso a estas tecnologías de la información y la comunicación (TIC), es parte de la agenda posible y deseada de nuevas culturas ciudadanas (Robles, 2009; Merino y Vega, 2011).

Este espacio público emergente está en sintonía con los modos del ejercicio ciudadano, o sea los vínculos y relaciones por medio de las redes sociales, ahora también como redes sociales virtuales, que organizan y concretan los procesos de comunicación entre ciudadanías en las diversas escalas sociales y aun geopolíticas. Manuel Castells (2000: 165-166) afirma en esta sintonía que la acción colectiva de la ciudadanía a través de las redes alojadas en internet permitirá impulsar procesos de reconstrucción del mundo actual, pero desde abajo, desde los pisos y espacios locales de las sociedades. Internet proporciona las bases materiales y tecnológicas que permiten el desarrollo de resistencias locales interesadas en la transformación de las injustas y desiguales sociedades actuales. Los riesgos en los propósitos de construir ciudadanías radicadas de modo exclusivo en las plataformas de comunicación/información también son latentes. La llamada ciberciudadanía tiene su contraparte en procesos de manipulación y 
sustitución de los espacios públicos virtuales por poderes locales y translocales, como puede ser la inducción y orientación del voto electrónico, por ejemplo (Pérez Luño, 2003). Sin olvidar los usos perversos de las esferas públicas virtuales que generalizan discursos del odio y propagan rumores falsos y juicios discriminatorios sin freno alguno.

Las culturas ciudadanas no pueden restringir su mirada de acción ni su utopía de sociedad a los contornos posibles y otorgados por instancias político-normativas de las formaciones estatales donde despliegan su existencia. Los nuevos espacios públicos accesibles a la participación de la ciudadanía son recursos necesarios, que requieren de procesos formativos-educativos que fomenten modos de acceso alternativo y propicien el uso libre y la apropiación permanente de los recursos y posibilidades que ofrecen las Tia. Es un reto que trasciende la tradicional formación cívica y ética que se da a los niños y niñas en las escuelas (Aguilera, 2010); tarea menuda, pero no imposible en contextos tan inequitativos como las actuales sociedades latinoamericanas (Eckholt y Lerner, 2009). Retos a la investigación y a la emergencia de nuevos campos de estudio se perfilan en torno a la llamada cibercultura y las diversas prácticas de los usuarios, entre las que se cuentan las desarrolladas con fines políticos por los ciudadanos/as que las utilizan (Escobar, 2005).

Hablar de culturas ciudadanas nos remite a procesos de formación cívica, a procesos de educación de la ciudadanía, a pedagogías ciudadanas diversas (González, 2012). En consonancia con lo anterior, también implica el desarrollo de habilidades y competencias comunicativas articuladas con las condiciones y los entornos comunicativos de la era actual (Valderrama, 2007: 226-227). Todo ello con la finalidad de construir ciudadanías capaces de reconocer los contextos culturales y sociopolíticos dentro de los cuales construyen su significación y su actuación política en las diversas escalas y niveles sociales del ejercicio ciudadano; en donde haya posibilidad de pensar utópicamente, porque la crítica y la acción política son indisociables de ciertas imágenes emergentes de la sociedad (Beck y Lemus, 2018: 14). Una ciudadanía que proponga alternativas de convivencia, opciones políticas para orientar el cambio y formas de intervención para solucionar los más importantes problemas que agobian a nuestras sociedades (Leyva et al., 2015; Stephen, 2016; Reguillo, 2017; Nasioka, 2017; Voces desde abajo, 2018; Durán y Moreno, 2018). Los resultados de estas iniciativas de acción ciudadana serán el desarrollo de prácticas y 
experiencias que modifican de forma profunda los marcos institucionales establecidos, los sistemas de creencias autoritarias y la jerarquía de valores que las sostienen (Calderón, Assies y Salman, 2002; Rodríguez, 2005; Baronnet, Mora y Stahler-Sholk, 2012; Sandoval, 2017).

Cultura + ciudadanía es por lo tanto enunciar un conjunto de prácticas sociales de los ciudadanos/as que modifican y reconfiguran los modos de estar, ser e interpretar los diversos hechos y acontecimientos en los que participan de un modo activo. Escribe Rafael Rodríguez que la ciudadanía es "un instrumento, una técnica de ejercicio de la democracia... no es un título de pertenencia; es el medio, la técnica, el instrumento que nos va a ayudar a construir los ámbitos de pertenencia y de acción” (2005: 175-176). Tener ciudadanía implica una concepción dinámica: se tiene para hacer algo, no sólo para verse reflejado en ella. Tiene además una cualidad creativa y defensiva, ya que conduce a la creación de relaciones tendientes al autogobierno y la emancipación y consolidación de espacios públicos abiertos y democráticos. La ciudadanía en este marco reflexivo no se considera, dice Rodríguez, "como un mero estatus ontológico o como receptáculo de una serie de derechos otorgados en el marco de un Estado-nación, más bien la ciudadanía ha de ser concebida desde un horizonte complejo que la redimensiona como un conjunto de procesos políticos, económicos y simbólicos que construyen lo real” (2005: 181).

La concepción liberal sobre la ciudadanía, que consiste en enfatizar el vínculo único y exclusivo entre el individuo y el Estado, ha dejado de ser la visión predominante para dar paso a una visión más abarcadora que la enriquece, ya que enfatiza no sólo las ciudadanías diferenciadas sino en particular la multilateralidad propiciada por los procesos de mundialización (Emmerich, 2009). La ciudadanía multilateral sería una conceptualización de mayor alcance explicativo sobre las transformaciones en los estados nacionales y en los vínculos que los individuos establecen con tales entidades políticas. Esta concepción de la ciudadanía multilateral cabe entenderla también como "la posibilidad de ser titular simultáneamente de varias ciudadanías ... poder ejercerlas con mayor o menor intensidad según los sentimientos de cada ciudadano hacia cada una de estas comunidades políticas" (Pérez Luño, 2003: 54). El éxito depende del desarrollo de una amplia cultura política ciudadana, que proporcione madurez y formación sólida a tales ciudadanos/as, que detentan, por ejemplo, una doble ciudadanía (Mateos, 2015). 


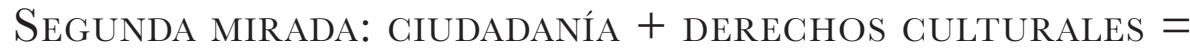
GIUDADANÍA CULTURAL

Transitar al binomio Ciudadanía + Cultura no sólo es desordenar los puntos de referencia y el orden de la relación conceptual, también es complejizar y abrir la discusión en consonancia con los cambios en las sociedades actuales, afectadas y cuestionadas por los procesos globales en sus diversas dimensiones: económica, política, tecnológica y cultural. Los procesos de inclusión y reconocimiento de nuevas ciudadanías son entonces asuntos actual y claramente vinculados con las reflexiones en torno a este binomio conceptual (Lachenal y Pirker, 2012).

En el contexto actual de la globalización, Jordi Borja (2010) expone varios elementos acerca de la ciudadanía que hay que destacar: "es un estatus, o sea, un reconocimiento social y jurídico por el que una persona tiene derechos y deberes de pertenencia a una comunidad casi siempre de base territorial y cultural. Los ciudadanos son iguales entre sí, y en teoría no se puede distinguir entre ciudadanos de primera, segunda, etc. En el mismo territorio, sometidos a las mismas leyes, todos tienen que ser iguales. La ciudadanía acepta la diferencia, no la desigualdad" (2010: 282-283). La ciudadanía tiene su acción y su desarrollo en contextos conflictivos, sean de confrontación o de diálogo social; está estrechamente vinculada a la democracia representativa y participativa; su escenario de acción privilegiado ha sido históricamente la ciudad. No hay progreso de la ciudadanía sin conflicto social y cultural con efectos políticos o jurídicos. La ciudadanía, concluye Borja, es "un concepto evolutivo, dialéctico: entre derechos y deberes, entre estatus e instituciones, entre políticas públicas e intereses corporativos o particulares. La ciudadanía es un proceso de conquista permanente de derechos formales y exigencias de políticas públicas para hacerlos efectivos" (2010: 285).

La conflictividad sociocultural y el dinamismo de los cambios experimentados por las ciudadanías en los procesos globalizadores dieron cabida a nuevas demandas (Norris, 2005). Por ejemplo, los procesos migratorios internacionales han impactado fuertemente las políticas de reconocimiento y de los derechos humanos de los inmigrantes, sean o no indocumentados. Crisis migratorias recientes como las procedentes de Centroamérica y del Caribe han cuestionado a los Estados nacionales y han abierto fuertes y permanentes debates sobre los derechos de las ciudadanías en tránsi- 
to y la criminalización y exclusión que experimentan estas poblaciones de migrantes (Ansley, 2007; Hernández et al., 2018).

Nuevos derechos surgieron en consonancia con los procesos de globalización en el último tercio del siglo xx que, de acuerdo con Pelfini, contribuyeron a "ampliar la ciudadanía más allá de los límites del Estado-nación: los derechos humanos, la protección del medio ambiente y el patrimonio cultural, entre otros, nos introducen en los difusos contornos del cosmopolitismo. Se trata de una nueva dimensión de la ciudadanía ...que alude a un elemento cultural o comunicacional" (2007: 25). Esta dimensión permitirá el acceso de la ciudadanía a un conjunto diverso de bienes culturales, a la preservación y expresión de su diversidad, y al tener mayor acceso a la información tendrán la capacidad para hacer valer su voz. Formar parte de la ciudadanía es así una cuestión de pertenencia, de adscripción identitaria, asunto no exento de conflicto y de una diversa y gran complejidad (Tamayo, 2010: 28-33).

De este modo, vinculado al complejo proceso de globalización, emergen los derechos culturales y comunicacionales, así como los derechos a la identidad que pueden expresarse a través del idioma, la historia y la tierra, junto con una serie diversa de derechos "conectivos" que se refieren al acceso y la participación en la industria cultural y en el amplio y complejo ámbito de la comunicación (León y Mora, 2006; Pelfini, 2007 : 28). Es así que en la actualidad se discute sobre la figura de la "ciudadanía digital" en relación con los derechos y obligaciones de las personas sobre las nuevas tecnologías de información y comunicación (Champeau e Innerarity, 2012; Adame, 2015). No sólo como individuos empoderados en sus capacidades tecnológicas y comunicativas, sino también como participantes en los diversos movimientos sociales contemporáneos que emplean de manera estratégica las tecnologías y las redes sociales (Adame, 2015: 123).

Se ha señalado que son los jóvenes los principales usuarios de estos soportes mediáticos como es el internet, plataforma de información y comunicación que ha impactado las prácticas de la ciudadanía (Reguillo, 2017; Padilla y Flores, 2011). El uso y la apropiación del internet ha propiciado la construcción de comunidades entre los mismos ciudadanos, orientadas por su pertenencia a diversas formaciones identitarias. Estas prácticas ciudadanas en la era del internet son comprensibles, considerando la dimensión cultural que permite reconocer las diversas identidades y marcos de acción compartidos. La transformación evidente se ubica en las prácticas 
ciudadanas en relación con el uso y sentido que se otorga a las nuevas plataformas de los medios digitales. El término de ciudadanía cultural nos conduce entonces y de modo natural a reconocer la diversidad de las prácticas y los nuevos sentidos asignados a los asuntos de interés público y de la esfera política. La consideración de la dimensión cultural de las prácticas políticas ciudadanas nos adentra en la consideración de las pertenencias y las adscripciones que entran en juego en las formas emergentes de participación en el espacio público y la arena de la política (Reguillo, 2003: 5). El internet, campo de acción de comunidades emergentes digitales, está anclada y afectada por las lógicas de la identidad y las formas culturales de las ciudadanías actuantes (Champeau e Innerarity, 2012). La proliferación de estas nuevas plataformas de las TIC no necesariamente potencian y fortalecen la praxis ciudadana; hay numerosa evidencia de que también resulta al contrario: desmovilización, desorientación, desinformación, circulación de discursos del odio, del miedo y el terror (casos como las cuentas falsas en twitter para apoyar el golpe de estado en Bolivia; las acciones de Cambridge Analytica en México, o la abundante propagación de crítica racista, de género, clasista y discriminadora en varios aspectos a favor y en contra de las acciones y políticas del régimen gobernante actual en México).

La mirada hacia los actores sociales en el ámbito mundial se ha transformado desde los años noventa, por lo menos. Todavía en los años setenta, la cuestión de la democracia y la participación ciudadana se centraba en torno a la discusión sobre los partidos políticos y los procesos electorales (Hall y Held, 1989; Heater, 2007). La lucha por el control del aparato estatal era crucial, la discusión sobre la vía y la estrategia para la toma del poder permeaban toda polémica y todo proyecto. Frente a los actores históricos como los campesinos y obreros, los otros actores sociales eran débiles o "invisibles". La sociedad tenía muy poco espacio de participación directa en ese marco de acción. En las democracias, la participación se reducía entonces a la competencia entre partidos políticos, a operar elecciones libres y procurar la libertad de prensa y opinión. Pero estos procesos de democratización de las sociedades dejaban de lado o asumían muy levemente impulsar la igualdad socioeconómica, el acceso a los bienes públicos y a la participación en las importantes decisiones colectivas. La ciudadanía se reducía a la esfera política y sin desarrollar o considerar los derechos sociales asociados (Pelfini, 2007: 30). Los deseos de auto- 
determinación y autoafirmación eran cuando mucho lejanas utopías. La necesidad de vincular los movimientos y acciones de la ciudadanía con el ámbito cultural se vislumbró como campo de acción política emergente, donde las disputas por la identidad cultural se tornaban necesarias (Laraña, 1999; Alonso et al., 1999; Cerda et al., 2011; Regalado, 2017).

Sin embargo, esto se ha ido transformando por la irrupción crítica, tanto en la teoría como en la práctica cotidiana, por parte de nuevos actores sociales como, por ejemplo, activistas de los movimientos por los derechos humanos, el feminismo, el ecologismo o bien las luchas de las comunidades y pueblos indígenas (Jelín, 1994; Curtin, 2002; Joppke, 2002; De la Peña, 2008; Cerda et al., 2011; Cerda, 2012; González Casanova, 2017). Para el caso de los latinos en los Estados Unidos de América, Renato Rosaldo se refiere al concepto de ciudadanía cultural como "el derecho de ser diferente (en cuanto a raza, etnia o lengua materna) frente a las normas de la comunidad nacional dominante, sin perjudicar el derecho de pertenecer, en el sentido de participar, en los procesos democráticos del Estado-nación" (1994: 67).

Frente al avance del neoliberalismo y el pensamiento tecnócrata, estas nuevas acciones colectivas aparecen como una alternativa para la democratización en los países de América Latina. Quizá una de las más interesantes respuestas de estas formas de organización ante la modernización y los procesos complejos de la globalización sea la creación de redes de relaciones sociales en diferentes niveles y dimensiones, tanto de escala local como de carácter internacional (Stennbergen, 1994; Riechmann y Fernández, 1994; Rubio, 2007; Borja, 2010; Stephen, 2016; Street, 2016).

En el ámbito internacional, desde hace más de veinte años han surgido y se han fortalecido redes de ayuda internacional de las potencias del Norte hacia los países del Sur, cuya intención es intervenir en contextos de exclusión económica y de opresión política. A pesar de que algunas de estas redes son asimétricas, puesto que las agencias financiadoras por lo general definen los temas y eligen a los receptores y canales de ejecución en el Sur, existen otras que manifiestan mayor reciprocidad, si no en términos del flujo de recursos sí de ideas y de prioridades. El campo de los derechos humanos y el mundo de las mujeres y la salud-enfermedad constituyen los ámbitos más extendidos, y los sigue el movimiento ambientalista, el de las poblaciones desplazadas y los procesos migratorios entre países y continentes (Eckholt y Lerner, 2009). Las redes nacionales 
e internacionales cuentan con una estructura organizativa desarrollada, con reglas de funcionamiento propias y una legitimidad progresiva frente a los gobiernos. Con frecuencia estas redes de organizaciones se convierten en voceras de las minorías discriminadas, representándolas frente al poder. Estos procesos pueden producir situaciones inesperadas, como la de cobijar movimientos democratizadores, o bien, al contrario, constituir una mera reproducción de formas paternalistas, populistas o autoritarias de relación entre las clases subordinadas y el poder (Casas y Carton, 2012; Lechenal y Pirker, 2012).

La expansión y el fortalecimiento de la ciudadanía es una tarea y un desafío para el proceso de consolidación de las democracias (Emmerich, 2009). La democracia, en una definición amplia, se considera "una forma de organización del poder que implica la existencia y buen funcionamiento del Estado; tiene en el régimen electoral un elemento fundamental, pero no se reduce a las elecciones; implica el ejercicio de una ciudadanía integral ...; es una experiencia histórica particular en la región, que debe ser entendida y valorada en su especificidad" (OEA y PNUD, 2010: 31-33). La democracia sería entonces una forma de organización del poder en la sociedad con el objetivo de ampliar permanentemente los derechos ciudadanos más allá de sus elementos básicos: civil, político y social, para expandirse e incluir las dimensiones económica y cultural.

El régimen democrático es el espacio donde se expresan y confrontan los diferentes proyectos sobre el orden social que se producen desde la sociedad civil. La democracia es un proceso de construcción permanente, hace participar a los ciudadanos, contribuye a generar una cultura de legalidad y al mismo tiempo el Estado de derecho (Cisneros, 2018: 25-26). Por ello, desde una perspectiva societaria, la consolidación de la ciudadanía implica el funcionamiento normal del Estado de derecho, que se expresa en la eliminación de las formas arbitrarias y del abuso del poder estatal y por la existencia de instituciones a las cuales se puede apelar para resolver conflictos sociales; significa además un control efectivo sobre las propias condiciones de vida y un cierto grado de previsión en la vida cotidiana (Jelín, 1994: 106). El Estado en el contexto actual no necesariamente va a promover la ampliación de la ciudadanía; parece que ahora ésta sólo puede ser promovida a través de actividades y demandas iniciadas y patrocinadas por organizaciones ciudadanas y movimientos de la sociedad civil (Casas y Carton, 2012; Bastos, 2012). 
Los actores sociales y los movimientos emergentes son sistemas colectivos de reconocimiento social, que expresan identidades colectivas, viejas y nuevas, con contenidos culturales y simbólicos importantes. Pero también son intermediarios políticos no partidarios que levantan las demandas de las voces no articuladas a la esfera pública y las vinculan con los aparatos institucionales del Estado. Esta función "expresiva" en la construcción de identidades colectivas y de reconocimiento social, así como su papel "instrumental", además de plantear un desafio a las estructuras institucionales existentes, también deberían ser vistos como "una garantía de un tipo de consolidación democrática que incluye un mecanismo de autoexpansión de sus fronteras y de autoperpetuación, que asegura una consolidación democrática dinámica" (Jelín, 1994: 106). Son movimientos que manifiestan un caudal de retos simbólicos frente al poder del Estado y a los procesos de hegemonía cultural prevalecientes.

Para el análisis de estas acciones colectivas emergentes es posible utilizar la noción de "redes de acción o movimiento", que se refiere al conjunto de grupos e individuos que comparten un escenario y cultura conflictivos y una identidad colectiva. Estas redes se caracterizan por: a) permitir una membresía múltiple, b) la militancia sólo parcial, c) el involucramiento personal y la solidaridad afectiva como requisito de participación, d) son grupos pequeños e inmersos en la vida cotidiana, conectados a través de ciertos conductos y redes sociales aparentemente invisibles. El aporte notorio de estas formas de acción colectiva es su contribución a la democratización de la vida cotidiana y a la creación de nuevos espacios públicos, así como al fortalecimiento de la sociedad civil y de su capacidad de autodeterminación. Estos fenómenos sociales constituyen realmente un mensaje, una afrenta o reto simbólico a los modelos socioculturales dominantes.

La relación entre cultura e identidad es directa, ya que en el centro de todo proceso cultural se encuentra la construcción de una identidad colectiva, dado que la cultura conforma la identidad de los grupos sociales al funcionar interiorizada en los sujetos como una lógica de las representaciones socialmente compartidas; dicha identidad se forma por referencia a un universo simbólico (Mata, Ballesteros y Gil, 2014). De modo que la identidad colectiva repercute en la reproducción y transformación de la cultura, por lo que al actuar como motor de la acción colectiva, uno de los efectos es la innovación cultural. El movimiento de las ciudadanías culturales expresa la constitución de cierta identidad colectiva que se asienta 
en una cosmovisión compartida, que se expresa en conductas y exteriorización simbólica, así como en la delimitación de oposiciones sociales más o menos definidas como el "nosotros" y el de uno o varios "ellos" (Miller, 2002; Nivón, 2014). La relación entre la ciudadanía y la alteridad se torna central tanto para reconocer procesos de construcción de identidades sociales como los retos y dificultades multidimensionales en el proceso de reconocimiento de las diferencias sociales y diversidades culturales (Castel, 2010: 287-300; Rubio, 2007: 93-95; Escalante, 2014: 227-230).

La ciudadanía cultural, de acuerdo con la Comisión Delors (UNEsco, 1999), orienta su accionar al desarrollo de procesos de autoformación y educación permanente en torno a cuatro ejes: 1) Aprender a Ser (el derecho a la autoidentificación y autodefinición); 2) Aprender a Conocer (el derecho a conocerse a sí mismo); 3) Aprender a Hacer (el derecho al autodesarrollo); 4) Aprender a Vivir Juntos (el derecho a la autodeterminación). Estos ejes para la acción de las ciudadanías efectivamente amplían y complejizan los modos de interrelación social y las formas de comunicación y representación política. Son una expansión de la diversidad, en franco distanciamiento de los procesos homogeneizadores e integracionistas de modelos de acción y representación hegemónicos. Esta demanda de reconocimiento a la diferencia también demanda la efectiva inclusión y participación en la vida democrática del país (García Canclini, 2004; Casas y Carton, 2012). La ciudadanía cultural se sostiene en la certeza de que no avanzará en sus reivindicaciones si no impulsa sus derechos a la cultura plena (Aguilera, 2010; Vich, 2014) y a una vida digna en la ciudad (Treviño y De la Rosa, 2009; Olvera y Olvera, 2015). La vía no sería la reproducción acondicionada y refuncionalizada de los modos degradados de la ciudadanía oficial masificada (Lomnitz, 2000), sino las formas y representaciones para la acción colectiva que orienten nuevos modos del buen vivir y experimentar la ciudad y el campo, como han sido los movimientos de reivindicaciones plurales de los pueblos indígenas y campesinos a lo largo y ancho de México (Baronnet, Mora y Stahler-Sholk, 2012; Bastos, 2012; Hernández y Martínez, 2013; Leyva, 2015).

\section{Consideraciones finales}

La relación entre ciudadanía y cultura es compleja, tal como se ha mencionado en los apartados arriba desarrollados y en las múltiples referencias utilizadas. Para finalizar esta revisión que intenta explorar las trans- 
formaciones actuales de la ciudadanía en sociedades como la nuestra, esquematizaré varios de los puntos expuestos. Al referirnos a las diversas y complejas prácticas de las "culturas ciudadanas" las hemos calificado con un conjunto de atributos y rasgos que las caracterizan, tales como los siguientes: que es una cultura adjetivada, que se ejerce sobre derechos reconocidos, tiene un dinamismo pragmático, sus habilidades son aprendidas en la interacción social, sus formas de competir están reglamentadas, inventa y retoma tradiciones de activismo social, exige lo otorgado por la ley, busca en su acción ser incluyente, su membresía es amplia aunque el individuo es el centro de su quehacer.

Por otra parte, al reflexionar sobre la emergencia de configuraciones de acción social de las denominadas como "ciudadanías culturales" podemos enumerar algunos rasgos sobresalientes: que son activas promotoras de proyectos y discursos orientados a la utopía; procuran la ampliación, definición y prosecución de nuevos derechos; su perfil de acción social es de múltiples dimensiones, con identidades flexibles y dinámicas; se perciben como colectividades en aprendizaje continuo, dado su perfil emergente, creando sus propias pedagogías orientadas a propugnar la innovación cultural y la generación de políticas públicas pertinentes a su mundo de vida, movilizadas por el reconocimiento, la igualdad y la diferencia; desarrollan esfuerzos por ampliar los contenidos de la ciudadanía, y la defensa del derecho a tener derechos. No obstante las diferencias y complementariedades, la dimensión cultural de las acciones ciudadanas toma de las disposiciones culturales lo que es estratégico para sus objetivos, y de la imaginación lo que no existe o está invisibilizado por las relaciones de poder y determinado por los contextos y específicas estructuras sociohistóricas. Hay un uso y una apropiación del saber acumulado y también la generación de experiencias para imaginar y desear nuevos derechos y posibilidades para la autogestión y autodeterminación como sujeto social, en un escenario en permanente transformación.

\section{BIBLIOGRAFÍA}

Abrahamian, Atossa Araxia (2015). The Cosmopolites: The Coming of the Global Citizen. Nueva York: Columbia Global Reports. https://doi.org/10.2307/j.cttlp6jhkj

Aceves, Jorge E. (2011). Nuevos movimientos sociales y ecologismo. México: CIEsas, Tesis de Doctorado en Ciencias Sociales. 
- (1997). "Ciudadanía ampliada. La emergencia de la ciudadanía cultural y ecológica", Razón y Palabra, vol. 1, núm. 5. Recuperado de Http://www.razonypalabra.org.mx/anteriores/n5/ciudad.htm

Adame Alemán, Juan Pablo (2015). Ciudadania digital: ¿oportunidad o amenaza? México: Cámara de Diputados, LXII Legislatura / Imagia Comunicación.

Aguilera Portales, Rafael Enrique (coord.) (2010). Educación para la ciudadanía. Monterrey: Comisión Estatal Electoral de Nuevo León.

Alonso, Jorge et al. (1999). El derecho a la identidad cultural. México: LVIICámara de Diputados

Ansley, Fran (2007). "Construyendo la ciudadanía sin licencia: la lucha de los inmigrantes indocumentados en Estados Unidos por el sustento y el reconocimiento", en Naila Kabeer (ed.), Ciudadanía incluyente. Significados y expresiones. México: UnAM-PUEG, pp. 209-226.

Arredondo Ramírez, Vicente (coord.) (2000). Ciudadanía en movimiento. México: Uia/Demos/IAPS.

- (1996). Hacia una nueva cultura ciudadana en México. Elementos para transformar a la sociedad mexicana. México: UIA-FAPRODE /Foro de Apoyo Mutuo.

Baronnet, Bruno, Mariana Mora y Richard Stahler-Sholk (coord.) (2011). Luchas "muy otras". Zapatismo y autonomía en las comunidades indígenas de Chiapas. México: UAM-Xochimilco / CiEsas / Universidad Autónoma de Chiapas.

Bastos, Santiago (coord.) (2012). Mezcala: la memoria y el futuro. La defensa de la isla en el bicentenario. México: CIESAS - Publicaciones de la Casa Chata.

Beck, Humberto y Rafael Lemus (ed.) (2018). El futuro es hoy. Ideas radicales para México. Madrid: Biblioteca Nueva.

Bonilla, Daniel (1999). La ciudadanía multiculturaly la política del reconocimiento. Santa Fe de Bogotá: Uniandes.

Borja, Jordi (2014). Revolución urbana y derechos ciudadanos. Buenos Aires: Café de las Ciudades.

- (2010). La ciudad conquistada. Madrid: Alianza.

Calderón, Marco, Willem Assies y Ton Salman (ed.) (2002). Ciudadanía, cultura política y reforma del Estado en América Latina. Zamora: El Colegio de Michoacán/IFe Michoacán.

Caletti, Sergio (2003). “Ciudadanía global o ciudadanía precarizada?”, en María Cristina Reigadas y Carlos A. Cullen (comp.). Globalización y nuevas ciudadanías. Mar del Plata: Suárez, pp. 85-114. 
Casas Guerrero, Rosalba y Hubert Carton de Grammont (comp.) (2012). Democracia, conocimiento y cultura. México: IIS-UnAM/Bonilla Artigas Editores.

Castel, Robert (2010). El ascenso de las incertidumbres: trabajo, protecciones, estatuto del individuo. Buenos Aires: Fondo de Cultura Económica.

Castells, Manuel (2009). Comunicación y poder. Madrid: Alianza.

(2000). La era de la información. Economía, sociedad y cultura, vol. I, La sociedad red. Madrid: Alianza.

- (2012, $1^{\circ}$ de septiembre). "El poder en la era de las redes sociales", Nexos. Disponible en: https://www.nexos.com.mx/?p=14970, consultado el 26 de junio de 2020.

Cerda García, Alejandro (2012). "Construyendo nuevas formas de ciudadanía. Resistencia zapatista en la región Altos de Chiapas", en Bruno Baronnet, Mariana Mora y Richard Stahler-Sholk (coord.), Luchas "muy otras". Zapatismo y autonomía en las comunidades indígenas de Chiapas. México: UAM-Xochimilco / CIESAS / Universidad Autónoma de Chiapas. , Anne Huffschmid, Iván Azura Monter y Stefan Rinke (ed.) (2011). Metrópolis desbordadas: poder, memoria y culturas en el espacio urbano. México y Berlin: UACM / CONACUlTA / Freie Universität Berlin.

Champeau, Serge y Daniel Innerarity (comp.) (2012). Internet y el futuro de la democracia. Barcelona: Paidós Ibérica.

Cisneros, Isidro H. (2018). "Política de la sociedad civil", El Punto Sobre la $i$, vol. 7, núm. 37, pp. 18-29.

Cortina, Adela (1997). Ciudadanos del mundo. Hacia una teoría de la ciudadanía. Madrid: Alianza.

Curtin, Deane (2002). "Ecological Citizenship" en E.F. Isin y B.S. Turner (ed.), Handbook of Citizenship Studie. Londres, Thousand Oaks y Nueva Delhi: SAGE Publications, pp. 293-304. https://doi.org/ 10.4135/9781848608276.n18

Durán, Inés y Rocío Moreno (coord.) (2018). Voces del México de abajo. Reflexiones en torno a la propuesta del CIG. Guadalajara: Cátedra Jorge Alonso - Universidad de Guadalajara / CIESAS.

Earls, Felton J. (2011). The Child as Citizen. Newbury Park, Londres y Nueva Delhi: Sage Publ.

Eckholt, Margit y Salomón Lerner Febres (ed.) (2009). Ciudadanía, democracia y derechos humanos. Quito: Abya-Yala.

Emmerich, Gustavo Ernesto (coord.) (2009). Situación de la democracia en México. México: Uam-Iztapalapa. 
Escalante Gonzalbo, Fernando (2014). "Ideas de la ciudadanía", en Enrique Florescano y José R. Cossío, Hacia una nación de ciudadanos. México: conaculta/FCE, pp. 211-231.

Escobar, Arturo (2005). "Bienvenidos a cyberia. Notas para una antropología de la cibercultura", Revista de estudios sociales, núm. 22, pp. 1535. https://doi.org/10.7440/res22.2005.01

Escobedo, Hernán y Angélica Camargo (2006). La investigación. Propuesta para la formulación de trabajos de grado en ciencias sociales. Bogotá: Universidad Piloto de Colombia.

Florescano, Enrique y José R. Cossío (coord.) (2014). Hacia una nación de ciudadanos. México: Conaculta/FGe.

Galindo, Jairo A. (2009). "Ciudadanía digital”, Signo y Pensamiento, vol. 28, núm. 54, pp. 164-173.

García Canclini, Néstor (coord.) (2004). Reabrir espacios públicos. Políticas culturales y ciudadanía. México: UAM-Iztapalapa /Plaza y Valdés.

(2001). "De la multiculturalidad a la ciudadanía global", en Francisco Blanco F. (ed.), Cultura y globalización. Colima: Universidad de Colima, pp. 125-142.

- (1999). La globalización imaginada. México, Buenos Aires y Barcelona: Paidós.

Garzón López, Pedro (2010). Ciudadanía indígena: del multiculturalismo a la colonialidad del poder. Madrid: Centro de Estudios Políticos y Constitucionales.

Gavilán, Iracema (2018). Movimientos culturales en defensa del territorio. Extractivismo y megaproyectos en el altiplano Wirikuta. Guadalajara: Cátedra Jorge Alonso - CIESAs / Universidad de Guadalajara.

González Ascencio, Gerardo (coord.) (2012). Cultura ciudadana y derechos humanos en México. México: conaculta.

González Casanova, Pablo (2017). Explotación, colonialismo y lucha por la democracia en América Latina. México: Akal.

González Luna, Teresa (1997). "Democracia y ciudadanía de género", La Ventana, Revista de Estudios de Género, núm. 5, pp. 230-241. https://doi. org/10.32870/lv.v0i5.366

Hall, Stuart y David Held (1989). "Citizens and Citizenship", en Stuart Hall y Martin Jacques (ed.), New Times: the Changing Face of Politics in the 1990s. Nueva York: Verso. 
Hannerz, Ulf (1998). Conexiones trasnacionales: cultura, gente, lugares. Madrid: Fronesis Cátedra / Universidad de Valencia.

Heater, Derek (2007). Ciudadanía. Una breve historia. Madrid: Alianza.

Hermes, Joke (2006). "Citizenship in the Age of the Internet", European Journal of Communication, vol. 21, núm. 3, pp. 295-309. https://doi. org/10.1177/0267323106066634

Hernández González, Eduardo y Paulina Martínez González (coord.) (2013). Participación ciudadana y desarrollo democrático. Guadalajara: Universidad de Guadalajara - Gu de la Ciénaga.

Hernández González, Eduardo (coord.) (2015). Exclusión y estrategias para una ciudadanía ampliada. Guadalajara: Universidad de Guadalajara Centro Universitario de la Ciénega.

Hernández López, Rafael A. et al. (2018). "Desplazamiento forzado de mujeres por Guadalajara”, en Inés Durán y R. Moreno (coords.). Voces del México de abajo. Reflexiones en torno a la propuesta del CIG. Guadalajara: Cátedra Jorge Alonso - Universidad de Guadalajara / CIESAS, pp. 205-236.

Instituto Nacional Electoral (INE) (2015). Informe País sobre la calidad de la ciudadanía en México. México: Instituto Nacional Electoral / El Colegio de México.

Jelín, Elizabeth (1994). “¿Ciudadanía emergente o exclusión? Movimientos sociales y ONG en los años noventa", Revista Mexicana de Sociología, vol. 56, núm. 4, pp. 91-108. https://doi.org/10.2307/3541084

Joppke, Christian (2002). "Multicultural Citizenship", en Engin F. Isin, y Bryan S. Turner (ed.). Handbook of Citizenship Studies. Londres, Thousand Oaks y Nueva Delhi: Sage Publications, pp. 245-258. https:// doi.org/10.4135/9781848608276.n15

Kabeer, Naila (ed.) (2007). Ciudadania incluyente. Significados y expresiones. México: UNAM / PUEG.

Krotz, Esteban y R. Winocur (2007). "Democracia, participación y cultura ciudadana: discursos normativos homogéneos versus prácticas y representaciones heterogéneas", Estudios Sociológicos, vol. xxv, núm. 73, pp. 187-218.

Kymlicka, Will (1996). Ciudadanía multicultural. Una teoría liberal de los derechos de las minorías. Barcelona: Paidós.

Lachenal, Gecile y Kristina Pirker (coord.) (2012). Movimientos sociales, derechos y nuevas ciudadanías en América Latina. México: Gedisa. 
Laraña, Enrique (1999). La construcción de los movimientos sociales. Madrid: Alianza.

Lechner, Norbert (2015). “¿Cuál es el imaginario ciudadano?”, en N. Lechner, Obras IV. Politica y subjetividad, 1995-2003. México: FCE / FLAGso, pp. 319-326.

- (2000). "Nuevas ciudadanías", Revista de Estudios Sociales, núm.5, pp. 25-31. https://doi.org/10.7440/res5.2000.03

León y Ramírez, Juan C. y Salvador Mora Velázquez (coord.) (2006). Ciudadanía, democracia y políticas públicas. México: FCSys-UnAM.

Leyva, Xóchitl et al. (2015). Prácticas otras de conocimiento(s). Entre crisis, entre guerras. Guadalajara: La Casa del Mago / Editorial Retos / PDTG, IWGiA, GALFisA y Proyecto Alice.

Linklater, Andrew (2007). Critical Theory and World Politics: Citizenship, Sovereignty and Humanity. Nueva York: Routledge. https://doi. org/10.4324/9780203960905

(2002). "Cosmopolitan Citizenship", en Engin F. Isin y Bryan S. Turner (ed.). Handbook of Citizenship Studies. Londres, Thousand Oaks y Nueva Delhi: sage Publications, pp. 317-332. https://doi. org/10.4135/9781848608276.n20

Lister, Ruth (2002). "Sexual Citizenship", en Engin F. Isin y Bryan S. Turner (ed.). Handbook of Citizenship Studies. Londres, Thousand Oaks y Nueva Delhi: SAge Publications, pp. 91-208.

Lomnitz, Claudio (2000). "La construcción de la ciudadanía en México", Metapolítica, vol. 4, núm.15, pp. 128-149.

Mata Benito, Patricia, Belén Ballesteros Velázquez e Inés Gil Jaurena (ed.) (2014). Aprendizaje de la ciudadanía y la participación. Madrid: Traficantes de Sueños.

Mateos, Pablo (2015). Ciudadanía múltiple y migración. Perspectivas latinoamericanas. México: CIESAS / CIDE.

Merino, José y A.F. Vega (comp.) (2011). Ciudadanos.mx, Twitter y el cambio político en México. México: Random House.

Miller, Toby (2002). "Cultural Citizenship", en Engin F. Isin y Bryan S. Turner (ed.). Handbook of Citizenship Studies. Londres, Thousand Oaks y Nueva Delhi: sage Publications, pp. 231-243. https://doi. org/10.4135/9781848608276.n14

Mockus, Antanas, Henry Murraín y María Villa (coord.) (2012). Antípodas de la violencia: desafios de cultura ciudadana para la crisis de (in)seguridad en 
América Latina. Washington: Banco Interamericano de Desarrollo / Corpovisionarios.

Moreno, Luis (2000). Ciudadanos precarios. La última red de protección social. Barcelona: Ariel Sociología.

Muñoz González, Germán (2008). "La ciudadanía juvenil como ciudadanía cultural: una aproximación teórica desde los estudios culturales", Revista Argentina de Sociología, vol. 6, núm. 11, pp. 217-236.

Nasioka, Katerina (2017). Ciudades en insurrección. Oaxaca 2006/Atenas 2008. Guadalajara: Cátedra Jorge Alonso-Universidad de Guadalajara/ CIESAS.

Natal, Alejandro; M. Benítez y G. Ortiz (coord.) (2014). Ciudadanía digital. México: uAM / Juan Pablos.

Nivón Bolán, Eduardo (2014) “Ciudadanía y cultura”, en Enrique Florescano y José R. Cossío, Hacia una nación de ciudadanos. México: conaCULTA / FCE, pp. 297-324.

Norris, Pipa (2005). "Global Governance and Cosmopolitan Citizens", en David Held y Anthony McGrew (ed.), The Global Transformation Reader. An Introduction to the Globalization Debate. Madden: Polity Press, pp. 287-297.

Olvera García, Jorge y Julio César Olvera García (coord.) (2015). Ciudad y ciudadanía. Hacia una resignificación desde el contexto mexicano. Toluca: UAEM / Porrúa.

Ong, Aihwa (2008). "Flexible Citizenship: The Cultural Logics of Transnationality", en Sanjeev Khagram y Peggy Levitt (ed.), The Transnational Studies Reader. Intersections \& Innovations). Londres y Nueva York: Routledge, pp. 446-458.

Ortiz Crespo, Santiago (2012). ¿Comuneros kichwas o ciudadanos ecuatorianos? La ciudadanía étnica y los derechos políticos. Quito: FLAcso.

Padilla De la Torre, María Rebeca y Dorismilda Flores Márquez (2011). "El estudio de las prácticas políticas de los jóvenes en internet", $\mathrm{Co}^{-}$ municación y Sociedad, Nueva Época, núm. 15, pp. 101-122.

Pelfini, Alejandro (2007). "Entre el temor al populismo y el entusiasmo autonomista. La reconfiguración de la ciudadanía en América Latina", Nueva Sociedad, núm. 212, pp. 22-34.

Peña, Guillermo de la (2008). "Derechos indígenas y ciudadanía étnica", en José L. Calva (coord.). Agenda para el desarrollo. Derechos y políticas 
sociales, vol. 12. México: UnAm / Porrúa / Cámara de Diputados LX Legislatura, pp.142-156.

Pérez Luño, Antonio Enrique (2003)._Ciberciudadania@ociudadan@.Com? Barcelona: Gedisa.

Programa de las Naciones Unidas para el Desarrollo y Secretaría General de la Organización de los Estados Americanos (2010). Nuestra democracia. México, FCE / OEA / PNUD.

Ramírez Saiz, Juan Manuel (2014). "Fundamentos de la construcción de ciudadanía mundial", en Enrique Florescano y José R. Cossío, Hacia una nación de ciudadanos. México: conaculta / FCE, pp.377-392.

- (2006). Ciudadanía mundial. Guadalajara, León y México: ITEso / UiA. Regalado, Jorge (coord.) (2017). Pensamiento crítico, cosmovisiones y epistemologías otras, para enfrentar la guerra capitalista y construir autonomías. Guadalajara: Cátedra Jorge Alonso - Universidad de Guadalajara / CIESAS. Reguillo, Rossana (2017). Paisajes insurrectos: jóvenes, redes y revueltas en el otoño civilizatorio. Barcelona y Guadalajara: Ned Ediciones / ITESO. (2003). "Ciudadanías juveniles en América Latina", Última Década, vol. 11, núm.19, pp. 1-20. https://doi.org/10.4067/S071822362003000200002

Reigadas, María Cristina y Carlos A. Cullen (comp.) (2003). Globalización y nuevas ciudadanías. Mar del Plata: Suárez.

Richardson, Diane (2001). "Extending Citizenship: Cultural Citizenship and Sexuality", en Nick Stevenson (ed.), Culture E' Citizenship. Londres, Thousand Oaks y Nueva Delhi: sage Publications, pp. 153166. https://doi.org/10.4135/9781446217665.n10

Richter Morales, Ulrich (2018). El ciudadano digital. Fake news y posverdad en la era del internet. México: Océano.

Riechmann, Jorge y Francisco Fernández (1994). Redes que dan libertad. Introducción a los nuevos movimientos sociales. Barcelona: Paidós.

Robles, José Manuel (2009). Ciudadanía digital: una introducción a un nuevo concepto de ciudadano. Barcelona: Universitat Oberta de Catalunya.

Rodríguez Prieto, Rafael (2005). Ciudadanos y soberanos. Participación y democracia directa. Madrid: Almuzara.

Rosaldo, Renato (2000). "La pertenencia no es un lujo. Procesos de ciudadanía cultural dentro de una sociedad multicultural", Desacatos. Revista de Antropología Social, vol. 2, núm. 3, pp. 39-49. 
- (1994). "Ciudadanía cultural en San José, California", en Néstor García Canclini et al., De lo local a lo global. Perspectivas desde la antropología. México: uAm-Iztapalapa, pp. 67-88.

Rubio Carracedo, José (2010). "De la ciudadanía posnacional a la ciudadanía transcultural”, en Rafael E. Aguilera Portales (coord.), Educación para la ciudadanía. Monterrey: Comisión Estatal Electoral Nuevo León, pp. 37-57.

- (2007). Teoría crítica de la ciudadanía democrática. Madrid: Trotta.

Safa, Patricia (2001). "Ciudadanía cultural y las identidades vecinales en las metrópolis: un acercamiento teórico", en Elsa Patiño y Jaime Castillo (comp.), Cultura y territorio, identidad y modo de vida. Puebla: RNIU, pp. 155-170.

- (1999). "Ciudadanía cultural y las organizaciones vecinales en la ciudad de México", en Fernando Carrión et al. (comp.), La ciudad, escenario de comunicación. Ecuador: FLACSO / ILDIS.

Sandoval, Rafael (coord.) (2017). Pueblos indígenas, creación de autonomía y revolución. Guadalajara: Cátedra Jorge Alonso - Universidad de Guadalajara / CIESAS.

Santiago Juárez, Rodrigo (2012). Lealtades compartidas. Hacia una ciudadanía multilateral. México: Plaza y Valdés.

Steenbergen, Bartvan (1994). "Towards a Global Ecological Citizen", en Bartvan Steenbergen (ed.), The Condition of Citizenship. Londres, Thousand Oaks y Nueva Delhi: sage Publications, pp. 149-151.

Stephen, Lynn (2016). Somos la cara de Oaxaca. Testimonios y movimientos sociales. México: Ciesas - Publicaciones de la Casa Chata.

Street, Susan (coord.) (2016). Con ojos bien abiertos: ante el despojo, rehabilitemos lo común. Guadalajara: Catedra Jorge Alonso.

Tamayo, Sergio (2010). Crítica de la ciudadanía. México: Siglo Xxi / uAM-Azcapotzalco.

Treviño Carrillo, Ana Helena y José Javier de la Rosa Rodríguez (2009). Ciudadanía, espacio público y ciudad. México: UACM.

Turner, Bryan S. (2001). "Outline of a General Theory of Cultural Citizenship", en N. Stevenson (ed.) (2001). Culture E Citizenship. Londres, Thousand Oaks, Nueva Delhi: sage Publications, pp. 11-32. https://doi.org/10.4135/9781446217665.n2

- (1994). "Post-Modern Culture/Modern Citizens", en Bartvan Steenbergen (ed.), The Condition of Citizenship. Londres, Thousand 
Oaks y Nueva Delhi: sage Publications, pp. 153-168. https://doi. org/10.4135/9781446250600.n12

UnEsco (1999). Ciudadanía cultural en el siglo XXI. Hamburgo: Instituto de la UNESCO para la Educación / CONFINTEA.

Valderrama, Carlos E. (2007). "Ciudadanía y educación del sujeto político en la sociedad de la información y el conocimiento", en Mónica Zuleta, Humberto Cubides y Manuel Escobar (ed.), ¿Uno o varios mundos? Diferencia, subjetividad y conocimientos en las ciencias sociales contemporáneas. Bogotá: Universidad Central / Siglo del Hombre / IESC, pp. 219-229. https://doi.org/10.4000/books.sdh.431

Vich, Víctor (2014). Desculturizar la cultura. La gestión cultural como forma de acción politica. Buenos Aires: Siglo XXI.

Voces desde Abajo y el equipo de Praxis en América Latina (2018). México: represión, resistencia y rebeldía: l@s zapatistas, el Congreso Nacional Indígena, lasmadresypadresde Ayotzinapa,l@snormalistas,l@s jornaler@s,l@s maestr@sdisidentes, las luchas por la autonomía y el territorio,otrosmovimientos sociales y la necesidad de una filosofia de la revolución. México: Juan Pablos.

Winocur, Rosalía (2002). Ciudadanos mediáticos: la construcción de lo público en la radio. Barcelona: Gedisa.

Zamorano, Gabriela (2008). “¿Desarrollando capacidades? Pobreza, "oportunidades" y ciudadanía neoliberal", Bajo el Volcán, vol. 7, núm. 12, pp. 67-88.

Forge Eduardo Aceves Lozano es profesor investigador de ciesas Occidente. Doctor en Ciencias Sociales. Líneas de investigación: antropología de la cultura e identidades urbanas, culturas del trabajo y populares; teoría y práctica de la historia oral y enfoque biográfico. Ha publicado libros individuales, colectivos y en revistas de investigación mexicanas, de Chile, Argentina, Brasil, España y Canadá. Libros recientes: G. de Garay y J. E. Aceves (coord.), Entrevistar ¿para qué? Múltiples escuchas desde diversos cuadrantes. México, Instituto Mora, 2017; J.E. Aceves, Uso de la historia oraly de vida en la investigación educativa. Aspectos metodológicos y fuentes orales. San Luis Potosí, El Colegio de San Luis, 2018 (Cuadernos del Centro). 
Ángela Renée de la Torre Castellanos

Directora de Encartes

Arthur Temporal Ventura

Editor

Verónica Segovia González

Diseño y formación

Cecilia Palomar Verea

María Palomar Verea

Corrección

Saúl Justino Prieto Mendoza

Difusión

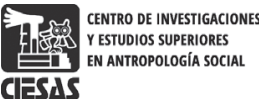

ClESAS
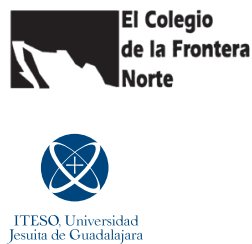

Equipo de coordinación editorial

Renée de la Torre Castellanos Directora de Encartes - Rodrigo de la Mora Pérez Arce ITeso - Arcelia Paz ciesas-Occidente - Santiago Bastos Amigo ciesas-Occidente Manuela Camus Bergareche Universidad de Guadalajara - Olivia Teresa Ruiz Marrujo El cOLEF - Christian Omar Grimaldo Rodríguez ITESO

\section{Comité editorial}

América Molina del Villar Directora general de ciesAS - Alberto Hernández Hernández Presidente de El cOLEF Enrique Páez Agraz Director del Departamento de Estudios Socioculturales del iтeso - Julia Esther Preciado Zamora CiESAS-Occidente - Subdirección difusión y publicaciones de CIESAS - Érika Moreno Páez Coordinadora del departamento de publicaciones de El colef - Manuel Verduzco Espinoza Director de la Oficina de Publicaciones del iteso - José Manuel Valenzuela Arce El colef • Luz María Mohar Betancourt Ciesas-Ciudad de México - Ricardo Pérez Monfort cIEsas-Ciudad de México • Sévérine Durin Popy CIESAS-Noreste • Carlos Yuri Flores Arenales Universidad Autónoma del Estado de Morelos • Sarah Corona Berkin DEcs/Universidad de Guadalajara - Norma Iglesias Prieto San Diego State University - Camilo Contreras Delgado El coleF • Alejandra Navarro Smith ITESO

Cuerpo académico asesor

Alejandro Frigerio

Universidad Católica

Argentina-Buenos Aires

Alejandro Grimson

usam-Buenos Aires

Alexandrine Boudreault-Fournier

University of Victoria-Victoria

Carlo A. Cubero

Tallinn University-Tallin

Carlo Fausto

UFrJ-Rio de Janeiro

Carmen Guarini

uBA-Buenos Aires

Caroline Perré

Centro de Estudios Mexicanos y

Centroamericanos-Ciudad de

México

Clarice Ehlers Peixoto

UERJ-Rio de Janeiro

\section{Claudio Lomnitz}

Columbia-Nueva York

Cornelia Eckert

UFrgs-Porto Alegre

Cristina Puga

unam-Ciudad de México

Elisenda Ardèvol

Universidad Abierta de

Cataluña-Barcelona

Gastón Carreño

Universidad de

Chile-Santiago

Gisela Canepá

Pontificia Universidad

Católica del Perú- Lima

Hugo José Suárez

unam-Ciudad de México

Jesús Martín Barbero

Universidad Javeriana-Bogotá
Julia Tuñón

INAH-Ciudad de México

María de Lourdes Beldi

de Alcantara

UsP-Sao Paulo

Mary Louise Pratt

NYU-Nueva York

Pablo Federico Semán

CONICET/UnSAM-Buenos Aires

Renato Rosaldo

NYU-Nueva York

Rose Satiko Gitirana Hikji

UsP-Sao Paulo

Rossana Reguillo Cruz

ITESO-Guadalajara

Sarah Pink

RMIT-Melbourne

Encartes, año 3, núm 6, septiembre 2020-febrero 2021, es una revista académica digital de acceso libre y publicación semestral editada por el Centro de Investigaciones y Estudios Superiores en Antropología Social, calle Juárez, núm. 87, Col. Tlalpan, C. P. 14000, México, D. F., Apdo. Postal 22-048, Tel. 548735 70, Fax 565555 76, encartesantropologicos@ciesas.edu.mx. Directora de la revista: Ángela Renée de la Torre Castellanos. Alojada en la dirección electrónica http://www.encartesantropologicos.mx. ISSN: 2594-2999. Las opiniones expresadas por los autores no necesariamente reflejan la postura de la revista. Se autoriza la reproducción parcial de los materiales publicados siempre y cuando se haga con fines estrictamente no comerciales y se cite la fuente. Salvo excepciones explicitadas, todo el contenido de la publicación está bajo una Licencia Creative Commons Atribución-NoComercial 4.0 Internacional. 